\title{
Colossal Malignant Nonteratoid Medulloepithelioma of the Eye with Unusual Metastases: The Natural History*
}

\section{Qureshi Sajid}

Assistant Professor, Department of Pediatric Surgical Oncology, Tata Memorial Center and Advanced Center for Treatment Research and Education in Cancer (ACTREC), Mumbai, Maharashtra, India

Correspondence: Qureshi Sajid, Asistant Professor, Department of Pediatric Surgical Oncology, Tata Memorial Hospital, E Borges Road, Parel, Mumbai 400012, Phone: 91-22-2417276, Fax: 91-22-24146937, e-mail: sajidshafique@rediffmail.com

\footnotetext{
Abstract

Background: Medulloepithelioma is rare intraocular tumor seen predominantly in children, and arising mainly from undifferentiated nonpigmented epithelium of the ciliary body.

Methods: We present a typical neglected case of malignant nonteratoid medulloepithelioma of the eye with extensive metastases to the facial soft tissue parotid and cervical lymph nodes.

Results: An aggressive surgical approach with orbital exenteration and comprehensive disease clearance with total parotidectomy and neck dissection augmented by postoperative radiotherapy was utilized after initial unsuccessful chemotherapy.

Conclusions: The present case highlights the natural history of untreated medulloepithelioma of the eye. Colossal growth at the primary site with extensive regional lymph node metastases develops without distant metastases. Complete surgical excision supplemented with postoperative radiotherapy should be considered even in the setting of advanced locoregional disease.

Keywords: Medulloepithelioma, nonteratoid, metastasis, natural history.
}

\section{INTRODUCTION}

Medulloepithelioma is a tumor of the primitive medullary epithelium overlying the ciliary body. ${ }^{1}$ Occasionally, it may arise from the optic nerve or the retina. Generally they are intraocular but can be locally aggressive. Medulloepithelioma rarely metastasizes, however, they can recur locally. The outcome for localized disease after treatment is good but the prognosis is worse when extraocular extension is present. ${ }^{2,3}$

This report documents a much delayed presentation of orbital medulloepithelioma with extensive metastases, which were successfully treated.

\section{CASE REPORT}

An 8-year-old boy presented with a fungating mass arising from the right eye. The swelling was small initially but it was neglected by the parents. The child was instead subjected to irrational aboriginal treatment over a period of one year. On examination, there was a $15 \times 10 \mathrm{~cm}$ exophytic mass arising from the right orbit (Figs 1A and B). In addition the child had a soft tissue deposit along the temporal region, a large parotid mass and multiple cervical lymph nodes. MRI revealed a large lobulated mass in the orbit encasing the proximal third of optic nerve with large nodal masses in the preauricular, parotid, submandibular and carotid spaces, displacing the carotid vessels (Figs 2A to C). Biopsy showed a nonteratoid medulloepithelioma. A CT scan of the thorax did not reveal any pulmonary metastases.

In view of the extensive locoregional disease chemotherapy (ifosfamide, cisplatin and etoposide) was commenced. However, after three cycles of chemotherapy there was no clinicoradiological change in the extent of disease. At this stage surgery was planned and an orbital exenteration with excision of the temporal fossa mass, total parotidectomy, and modified neck dissection was performed (Figs 3A to D). The patient had an uneventful postoperative recovery. He also received radiotherapy (50.4 Gy) to the right side of face and neck.

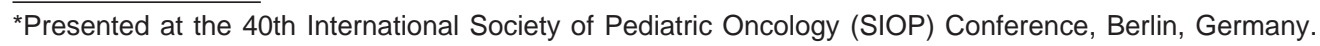




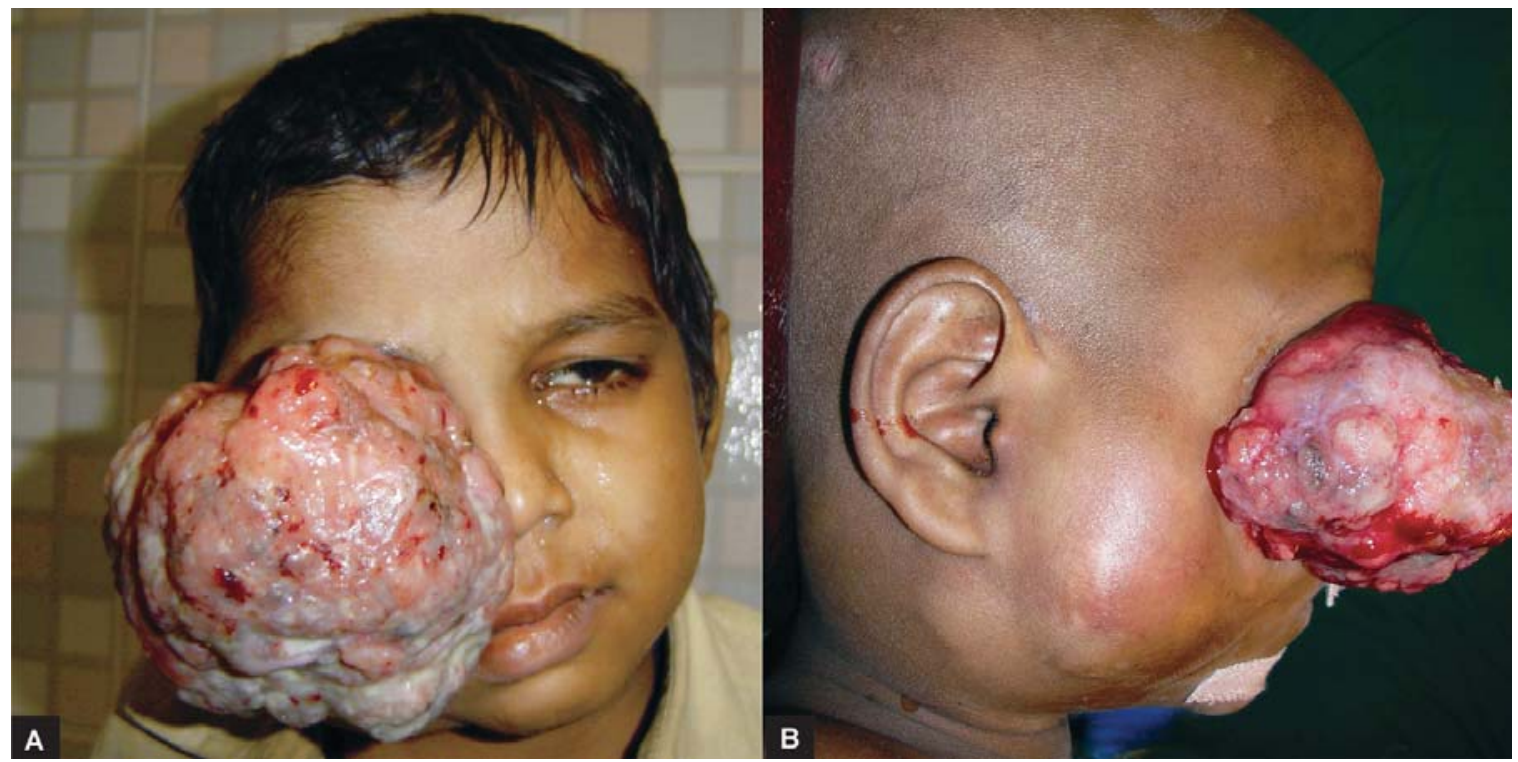

Figs $1 \mathrm{~A}$ and $\mathrm{B}$ : Clinical photograph showing the extent of locoregional disease
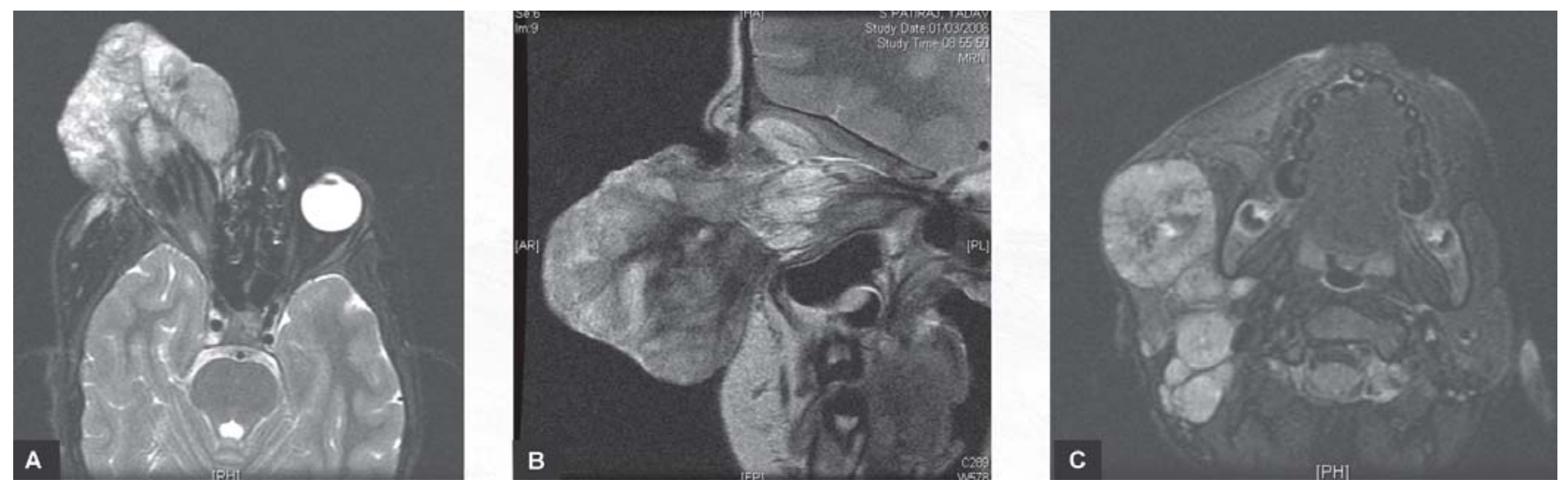

Figs $2 \mathrm{~A}$ to $\mathrm{C}: \mathrm{MRI}$ showing the orbital mass and the parotid and cervical lymph nodes

Histopathology revealed cords and trabeculae of neuroepithelium with intervening glial and retinal type tissue (Figs 4A and B). Neuroepithelium was S100, NSE, EMA, Cytokeratin, and HMB45 positive. Glia was GFAP positive.

An artificial external orbital prosthesis provided acceptable cosmesis (Figs 5A to C). At three year followup the patient is alive and disease-free.

\section{DISCUSSION}

Most patients with medulloepithelioma present with visual symptoms, pain, protrusion of the eye, cataract, glaucoma, lens coloboma, etc. ${ }^{1-3}$ Presentation with a colossal fungating primary lesion with extensive regional disease is very rare. The reason for the significant delay in seeking medical attention in our case may be numerous and which perhaps are prevalent in most developing countries. They include poverty, ignorance, illiteracy, lack of basic medical facilities and most importantly the unshakable faith in local treatment remedies. Our patient was subjected to traditional irrelevant therapy which led to significant delay and resultant massive growth of the primary and metastases to the parotid, cervical lymph nodes and facial soft tissue. This unique but regrettable delayed presentation provided the opportunity to observe the natural history of untreated medulloepithelioma. However, despite such extensive locoregional disease there were no distant metastases to any solid organs, and the lesion appeared technically resectable on imaging.

The best treatment for medulloepithelioma is uncertain. Due to its rarity, acquiring enough experience in different 


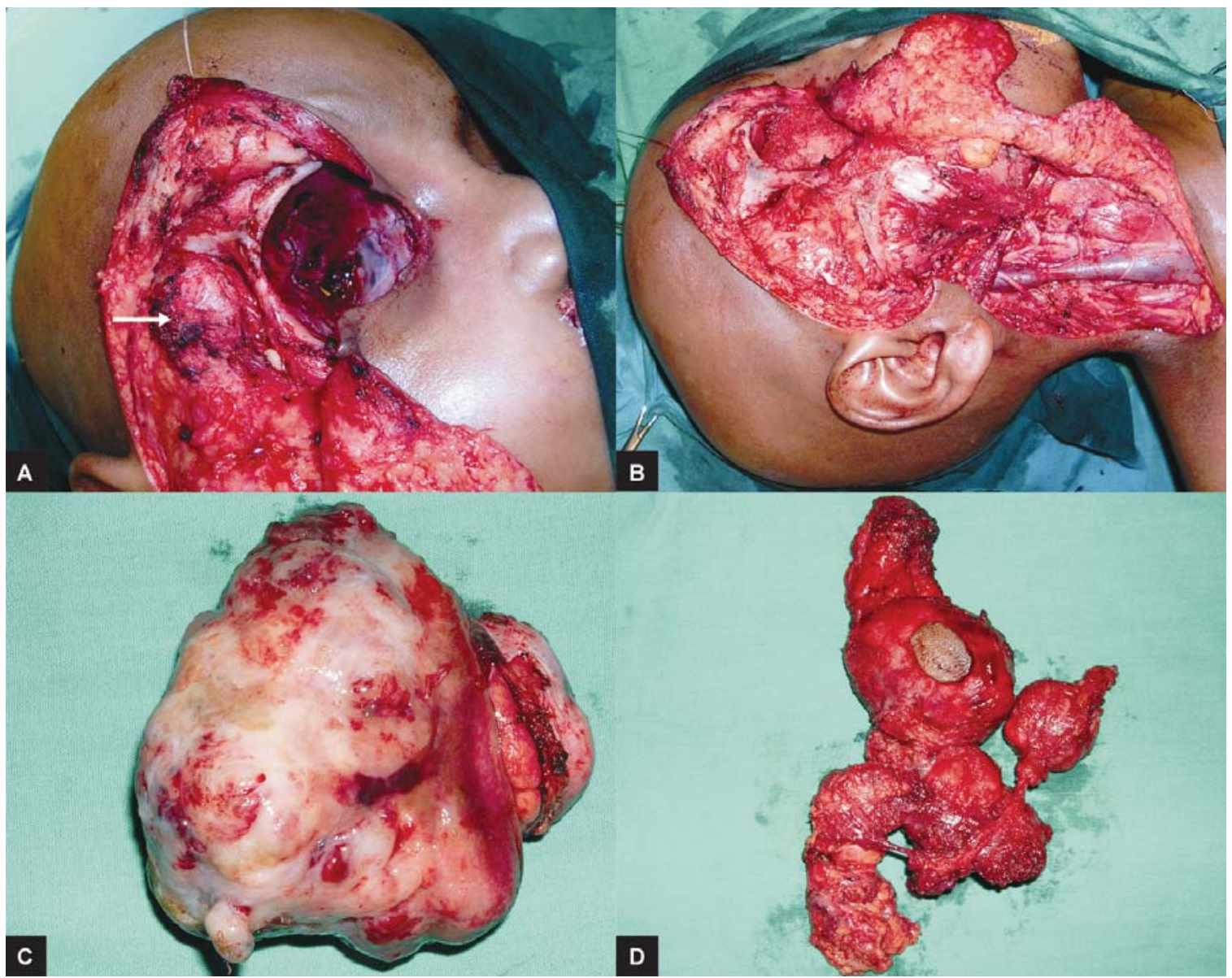

Figs 3A to D: Intraoperative photograph after exenteration, total parotidectomy and modified neck dissection. Note the nodule in the temporal fossa (arrow)

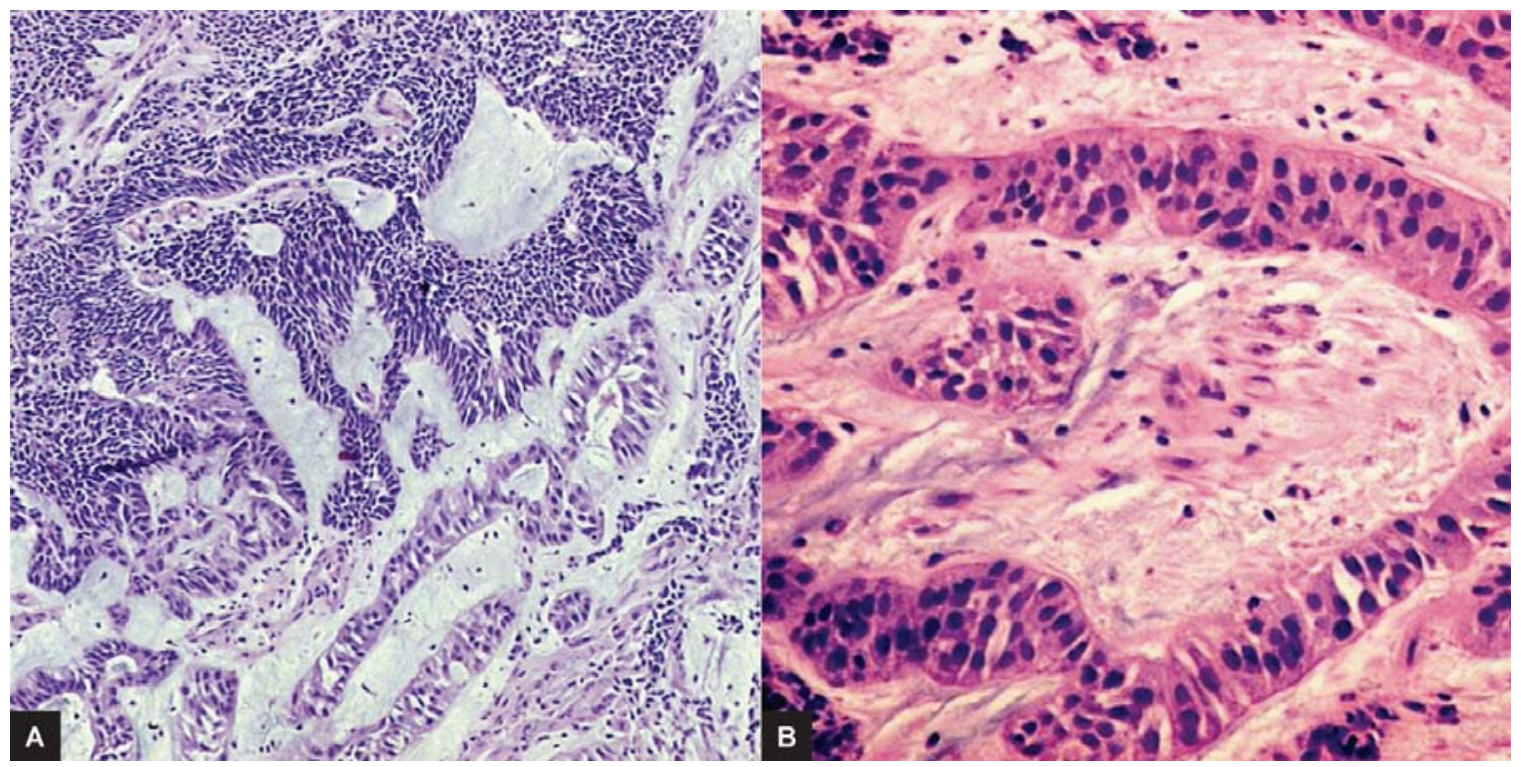

Figs 4A and B: Microphotograph showing neuroepithelium with retinal differentiation $\mathrm{H}$ and $\mathrm{E}$ 

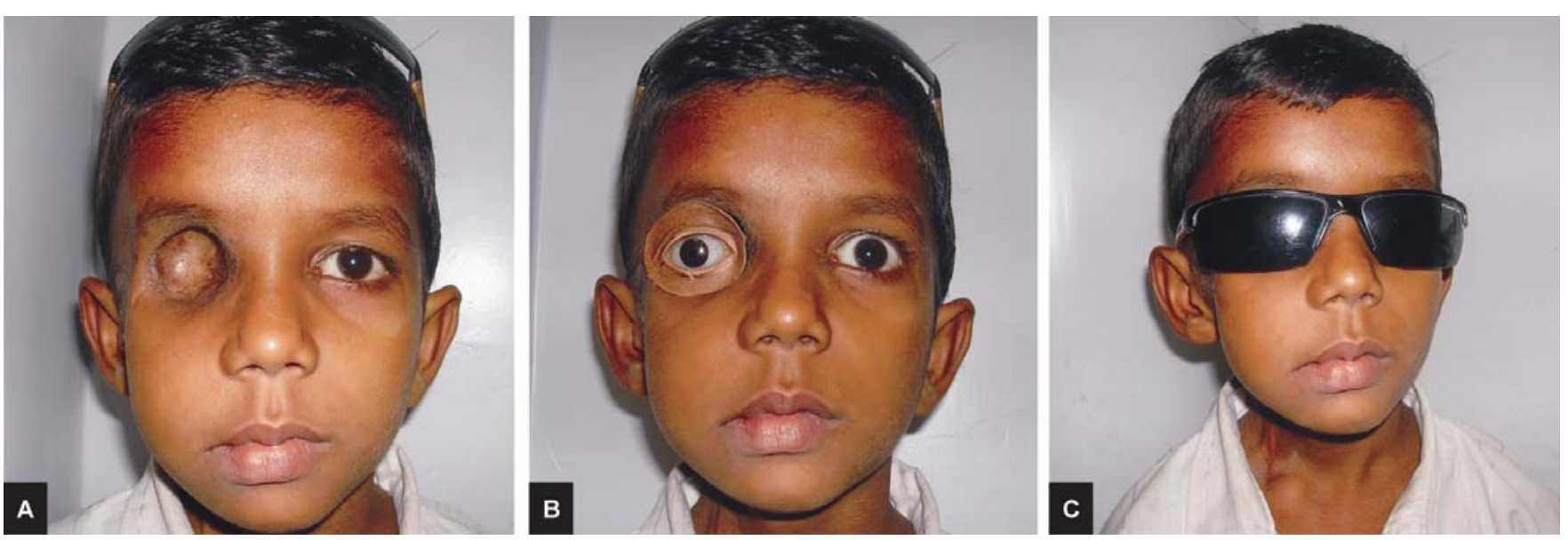

Figs 5A to C: Postoperative clinical photographs

therapeutic strategies for any one center is difficult. For conventional medulloepithelioma enucleation is the treatment of choice, however, local excision has also been performed albeit with the obvious risk of local recurrence. ${ }^{1-3}$ The use of local irradiation (scleral plaque or proton beam) or chemotherapy has not been established; it is only recommended for local recurrence or distant metastases. ${ }^{4,5}$

The treatment strategy and clinical outcomes of extensive medulloepithelioma with metastases have not been previously assessed. In view of the large size and extensive metastases chemotherapy was initially administered which however did not produce any appreciable change in the tumor. An aggressive surgical approach with orbital exenteration and comprehensive disease clearance with total parotidectomy and neck dissection augmented by postoperative radiotherapy was utilized. The patient is well rehabilitated and continues to be disease free at three years follow-up.

\section{CONCLUSION}

The present case highlights the natural history of untreated medulloepithelioma of the eye. Colossal growth at the primary site with extensive regional lymph node metastases develops without distant metastases. Complete surgical excision supplemented with postoperative radiotherapy should be considered even in the setting of advanced locoregional disease. The role of chemotherapy is uncertain.

\section{REFERENCES}

1. Broughton W, Zimmerman L. A clinicopathologic study of 56 cases of intraocular medulloepitheliomas. American Journal of Ophthalmology 1978;85:407-18.

2. Canning CR, McCartney AC, Hungerford J Medulloepithelioma (diktyoma). Br J Ophthalmol 1988;72:764-67.

3. Shields JA, Eagle RC Jr, Shields CL, Potter PD. Congenital neoplasms of the nonpigmented ciliary epithelium (medulloepithelioma). Ophthalmology 1996;103:1998-2006.

4. O’Keefe M, Fulcher T, Kelly P, Lee W, Dudgeon J. Medulloepithelioma of the optic nerve head. Archives of Ophthalmology 1997;115:1325-27.

5. Davidorf FH, Craig E, Birnbaum L, Wakely P Jr. Management of medulloepithelioma of the ciliary body with brachytherapy. Am J Ophthalmol 2002;133:841-43. 